\title{
DESIGN AND DEVELOPMENT OF FERROFLUID BASED RECONFIGURABLE ANTENNA
}

\author{
Rocktotpal Baruah $^{1}$, Nidhi S. Bhattacharyya ${ }^{2}$ \\ ${ }^{I}$ Microwave Engineering Laboratory, Department of Physics, Tezpur University \\ ${ }^{2}$ Microwave Engineering Laboratory, Department of Physics, Tezpur University
}

\begin{abstract}
An antenna resonant frequency reconfiguration mechanism based on colloidal solution of magnetic nanoparticles (ferrofluid) is presented. The property of magnetically controllable flow of ferrofluid is the key parameter in this aspect. The method to reconfigure the antenna resonating frequency is relies on the changes in the dielectric constant of structurally modified antenna substrate material. Presence of micro channels, partially filled with ferrofluid offers a spatial variation of dielectric constant. A controlled spatial variation of dielectric constant leads to a user defined frequency reconfigurability, supported by the scattering $S_{11}$ parameter study. Antenna radiation pattern, gain and efficiency measurements are carried out to study the antenna performances at all the reconfigured frequencies.
\end{abstract}

Keywords: reconfigurable antenna, micro channel, ferrofluid, microstrip patch antenna

\section{INTRODUCTION}

Along with other areas, nano structured materials have found their potential utilizations in planar microwave antennas. Recently, in various approaches nano structured materials have been used in applications like low profiling and broad banding of planar antennas. In literature [1]antenna miniaturization is achieved by using ferrite nanoparticlesas filler material for the antenna substrate. In another approach [2]metallic nanoparticles basedinkjet printed antennawith high gain is investigated. Tune ability of material parameters nano sized particles through the application of external fields catch the attraction of investigators towards another aspect,called smart material. Smart materials possess the property to change material parameters like permittivity, permeability etc. and are normally used as antenna substrates in reconfigurable antennas to change antenna operating characteristics. Reconfigurable antennas are basically a type of antenna which offers tune ability of antenna operating frequency, radiation pattern and polarization [3]. Due to their attractive operational flexibility and compactness planarfrequency reconfigurable antennas are of great importance in multi standard wireless communications. Apart from the smart materials, frequency reconfiguration is done by implicating various electrically controlled switching elements viz. RFMEMS, PIN diodes, Varactors etc.[4-6], optically controlled switches [7] and mechanically tuned structures[8]. However, these methods possess some disadvantages like non-linearity of the switching elements, high biasing voltages and interference due to the biasing lines, complicated activation mechanism and bulk design[3]. In substrate material based tuning, frequency reconfiguration is mostly done by altering the dielectric constant of the substrate material, which plays an important role in antenna operating characteristics and the technique is less prone to the effects of interference and non-linearity[3, 9].
The work presents a frequency reconfigurable planar antenna based on modified substrate material. In this approach substrate modification is done by placing micro channel beneath the antenna radiating patch which is partially filled with ferrofluid (FF). As the position of ferrofluid within the channel can be controlled with the help of an external magnetic field; dielectric constant of the substrate material beneath the radiation can altered. The method uses two electromagnets to control the ferrofluid position and hence thespatial variations of the dielectric constant of the substrate.This spatial variation of the dielectric constant results a shifting of the antenna operating frequency.

\section{ANTENNA DESIGN}

The proposed antenna is basically a microstrip patch antenna designed to operate at $8 \mathrm{GHz}$, using Transmission Line Model (TLM) method. The antenna is fabricated on a glass epoxy (FR4) substrate of thickness $1.6 \mathrm{~mm}$. An inverted L shaped glass micro channel of inner diameter $0.9 \mathrm{~mm}$ is placed into the substrate along the edge of the radiating patch, as shown in Figure 1. The channel is half filled with ferrofluid which is synthesised using the procedures mentioned in [10]. Two electromagnets are attached to the ground plane of the antenna along the two arms of the $\mathrm{L}$ shaped channel and are separately connected to an external power source. 
(a)

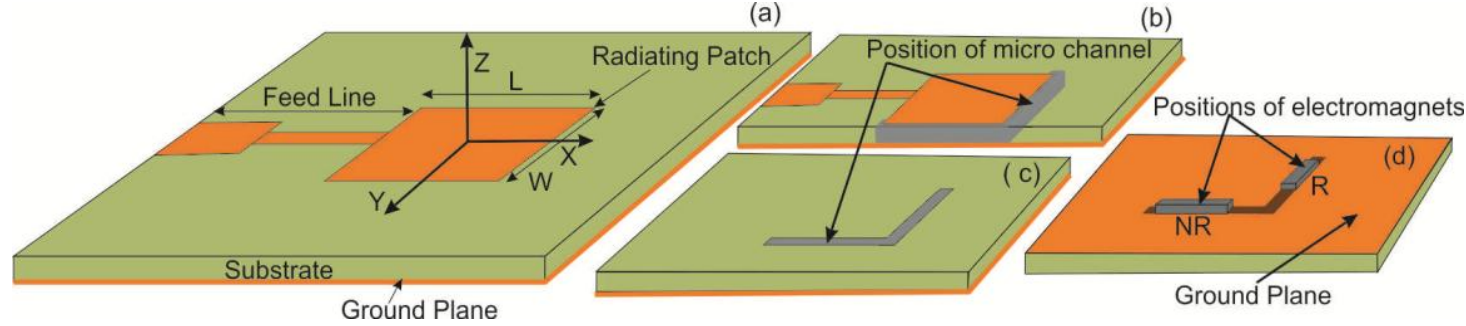

Ground Plane

Fig 1: (a) Schematic diagram of the antenna, (b) Cross sectional view, (c) Top view and (d) Backside view with the positions of electromagnets

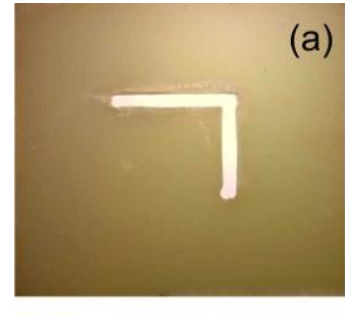

(b)

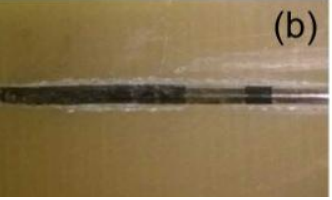

Fig 2: Fabricated antenna (a) Slot for micro channel, (b) Micro channel with FF, (c) Final prototype antenna

\section{MATERIAL SYNTHESIS}

Ferrofluid is synthesised in a process of two steps. First process involves the synthesis of magnetic nanoparticles of preferred dimensions. In in this approach magnetite (Fe3O4) nanoparticles are synthesized by co-precipitation technique [10]. Precursor solutions of $\mathrm{FeCl} 3$ and $\mathrm{FeCl} 2$ are taken in the molar ratio of 0.5 and then added concurrently to a $0.4 \mathrm{~N}$ $\mathrm{HCl}$ solution of volume $25 \mathrm{ml}$. A $1.5 \mathrm{~N}$ solution of $\mathrm{NaOH}$ is prepared and added to the resulting solution under constant stirring while maintaining the $\mathrm{pH}$ of the final solution at 12 . The process resulted in a dark black precipitate. Impurity free precipitate of magnetic nanoparticles are obtained after repeated washing and centrifugation. In order to stop further oxidation a $0.01 \mathrm{~N} \mathrm{HCL}$ is added to the sample.

In the second step synthesized magnetic nanoparticles are coated with surfactant layer. Approximately $400 \mu 1$ of anionic surfactant oleic acid [C18H34O2, 99\% pure, Otto] are added to the sample under vigorous stirring. Finally, these surfactant coated nanoparticles are dispersed to ARgrade methanol in a concentration of $\sim 6.6 \%(\mathrm{wt} / \mathrm{vol})$.

\section{RESULT AND DISCUSSION:}

\subsection{Microstructural Study of Fe3O4 Nanoparticles}

The microstructure of oleic acid coated nanoparticles is studied by an X-ray diffractometer (Rigaku MiniFlex 2000). Figure 3 shows X-ray diffractrogram of the nanoparticles and the pattern exhibit (220), (311), (511) and (440) diffraction peak of $\mathrm{Fe} 3 \mathrm{O} 4$. The plans are determined from JCPDS card no 65-3107.

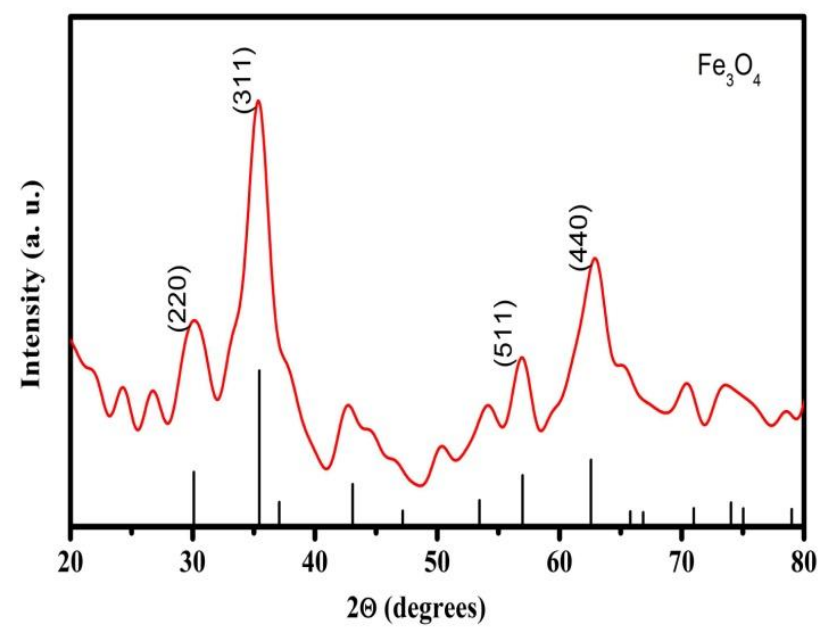

Fig 3: XRD difractogram of Fe3O4 (FFT smoothed)

\subsection{Antenna Performance Study}

Based on the proposed idea aprototype antenna is fabricated and experimental studies are carried for S11 parameters against frequency, antenna radiation pattern, antenna gain and efficiency. All the measurement are done by using Agilent VNA () and antenna measurement system of Diamond Engineering, U.S.A.

\subsubsection{S11 Parameters}

The measured S11 parameters of the designed antenna are shown in Figure 3. While measuring the S11 parameters four different conditions are taken into consideration, namely the original antenna designed for operating at 8 $\mathrm{GHz}$, antenna with the micro channel, antenna with halffilled micro channel and positioning ferrofluid in two different positions $\mathrm{R}$ and $\mathrm{NR}$ as illustrated in Figure 1. Figure 4 shows shifting of $-10 \mathrm{~dB}$ bandwidth in all the four cases which is mainly due to the changes in the effective dielectric constant of substrate material under the radiating patch. Among the four cases last two cases are considered for electromagnetically tune the antenna operating frequency. The entire volume of ferrofluid in the micro channel can be repositioned to the positioned marked as $\mathrm{R}$ and NR by activating the respective electromagnets attached to the position. With the repositioning of ferrofluid there is a spatial alternation of dielectric constant of the substrate under the radiating patch, which leads to the reconfiguration of the antenna operating frequency. The designed antenna offers a frequency shifting of $\sim 400 \mathrm{GHz}$. At position $\mathrm{R}$ the 
antenna operates at $7.45 \mathrm{GHz}$ while in position NR antenna operating frequency changes to $7.85 \mathrm{GHz}$.

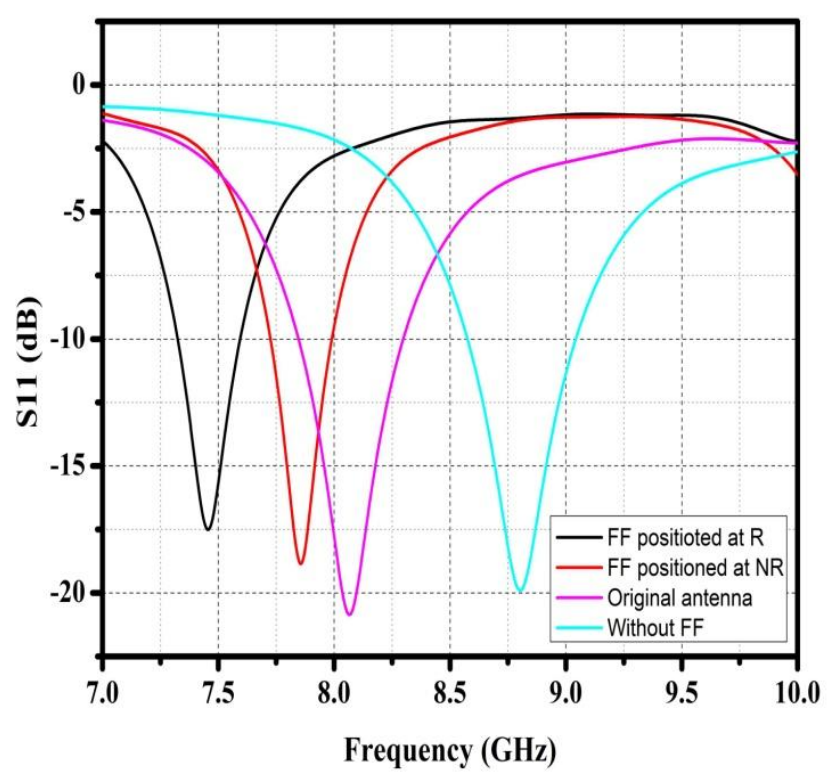

Fig 4: S11 parameters of the antenna for four different conditions

\subsubsection{Radiation Pattern}

The radiation pattern of the prototype antenna is measured for both the position R and NR and co and cross polarization radiation patterns are shown in Figure 5 (a) and (b) respectively. At position $\mathrm{R}$ the antenna radiates in a broad sight radiation pattern of directivity $6.99 \mathrm{dBi}, 3 \mathrm{~dB}$ beam width $72.48^{\circ}$ and main lode direction $10.12^{\circ}$ and at NR position radiation pattern is almost similar to the previous case with directivity $7.8 \mathrm{dBi}, 3 \mathrm{~dB}$ beam width $72.78^{\circ}$ and main lobe direction $11.42^{\circ}$. From the measured result, it is observed that the prototype antenna radiates in almost identical patterns at reconfigured operating frequencies. The antenna also offers a good cross polar isolation of minimum $-10 \mathrm{~dB}$ at both the operating modes.

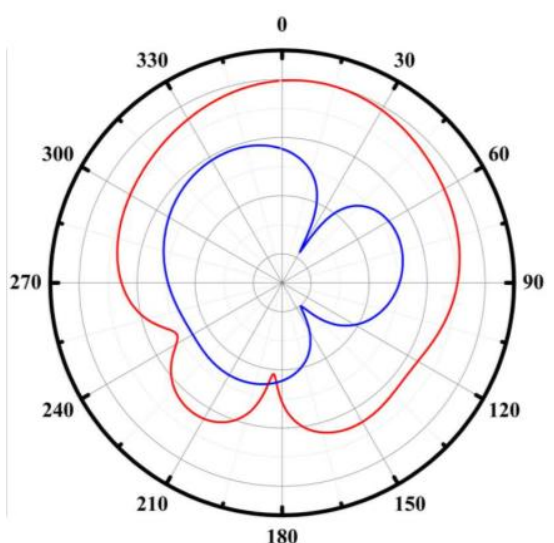

(a)

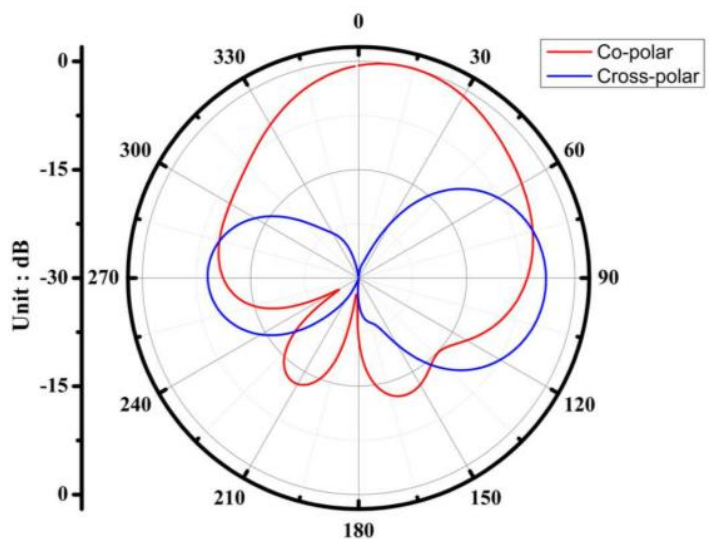

(b)

Fig 5: Antenna radiation patterns of (a) position $R$, (b) position NR

\subsubsection{Gain and Efficiency}

The measured gain and efficiency of the antenna at both the accessible frequencies are given in Table 1. At frequency $7.45 \mathrm{GHz}$ the antenna radiates with a gain of $4.89 \mathrm{dBi}$ and efficiency of $69.95 \%$. The antenna also exhibits comparatively constant gain and efficiency of $5.24 \mathrm{dBi}$ and $67.17 \%$ at $7.85 \mathrm{GHz}$. The observed result shows that the antenna can reconfigured to both the frequencies without any significant losses in the signal power.

Table 1: Antenna gain and efficiency

\begin{tabular}{|c|c|c|c|}
\hline Position & $\begin{array}{c}\text { Frequency } \\
(\mathrm{GHz})\end{array}$ & $\begin{array}{c}\text { Gain } \\
(\mathrm{dBi})\end{array}$ & $\begin{array}{c}\text { Efficiency } \\
(\%)\end{array}$ \\
\hline R & 7.45 & 4.89 & 69.95 \\
\hline NR & 7.85 & 5.24 & 67.17 \\
\hline
\end{tabular}

\section{CONCLUSION}

The work presents an antenna operating frequency reconfiguration technique which is based on modified substrate material. Antenna substrate is modified by placing micro channels half filled with ferrofluid in it. The main concept of frequency reconfiguration lies on spatial alternations of dielectric constant of the substrate under the radiating patch. The desired dielectric constant variation is achieved by electromagnetically controlled repositioning of ferrofluid. With the alterable dielectric constant the prototype antenna can be reconfigured to two different frequencies $7.45 \mathrm{GHz}$ and $7.85 \mathrm{GHz}$. The design antenna exhibits a stable radiation pattern of $3 \mathrm{~dB}$ beamwidth of $72.00^{\circ}$ and main lobe direction of $\sim 10.00{ }^{\circ}$ at all reconfigured frequencies. Comparatively constant gain and efficiency are also realized by the antenna at these 
frequencies. From the observed results it can be concluded that the proposed antenna can effectively reconfigure its operating frequency between two values. The technique is free from some of the major issues such as non-linearity, high biasing voltage, interference due biasing lines and complicated structure etc. possess by other reconfiguration techniques like electrical, optical and mechanical. The proposed antenna may find potential application in multi standard wireless communications devices.

\section{REFERENCES}

[1] J. L. Mattei, L. Huitema, P. Queffelec, J. F. Pintos, P. Minard, A. Sharahia, et al., "Suitability of Ni-Zn Ferrites Ceramics With Controlled Porosity as Granular Substrates for Mobile Handset Miniaturized Antennas," IEEE Transactions on Magnetics, vol. 47, pp. 3720-3723, 2011.

[2] B. S. Cook and A. Shamim, "Inkjet Printing of Novel Wideband and High Gain Antennas on Low-Cost Paper Substrate," IEEE Transactions on Antennas and Propagation, vol. 60, pp. 4148-4156, 2012.

[3] C. G. Christodoulou, Y. Tawk, S. A. Lane, and S. R. Erwin, "Reconfigurable antennas for wireless and space applications," Proceedings of the IEEE, vol. 100, pp. 2250-2261, 2012.

[4] L. Ge and K. M. Luk, "Frequency-Reconfigurable Low-Profile Circular Monopolar Patch Antenna," IEEE Transactions on Antennas and Propagation, vol. 62, pp. 3443-3449, 2014.

[5] T. Li, H. Zhai, X. Wang, L. Li, and C. Liang, "Frequency-Reconfigurable Bow-Tie Antenna for Bluetooth, WiMAX, and WLAN Applications," IEEE Antennas and Wireless Propagation Letters, vol. 14, pp. 171-174, 2015.

[6] J. Chang won, L. Ming-jer, G. P. Li, and F. D. Flaviis, "Reconfigurable scan-beam single-arm spiral antenna integrated with RF-MEMS switches," IEEE Transactions on Antennas and Propagation, vol. 54, pp. 455-463, 2006.

[7] C. J. Panagamuwa, A. Chauraya, and J. C. Vardaxoglou, "Frequency and beam reconfigurable antenna using photoconducting switches," IEEE Transactions on Antennas and Propagation, vol. 54, pp. 449-454, 2006.

[8] S. J. Mazlouman, M. Soleimani, A. Mahanfar, C. Menon, and R. G. Vaughan, "Pattern reconfigurable square ring patch antenna actuated by hemispherical dielectric elastomer," Electronics Letters, vol. 47, pp. 164-165, 2011.

[9] L. Liu and R. J. Langley, "Liquid crystal tunable microstrip patch antenna," Electronics Letters, vol. 44, pp. 1179-1180, 2008.

[10] M. Devi and D. Mohanta, "Rheological Properties of Iron Oxide Based Ferrofluids," in TRANSPORT AND OPTICAL PROPERTIES OF NANOMATERIALS: Proceedings of the International Conference-ICTOPON-2009, 2009, pp. 495-501. 